\title{
Application Research on 2D Image-based Non-contact Anthropometric Technology in Clothing E-commerce
}

\author{
Shunhua Luo \\ School of fashion design, Shandong University of Art and Design, Jinan Shandong 250300, China \\ Ish052002@163.com
}

\begin{abstract}
Anthropometry is an important component element of fashion design, pattern research, clothing production and clothing MTM online. Anthropometric technology has went through the process from contact to non-contact, and from manual anthropometry to 2D image and 3D anthropometry. The purpose of this paper is to summarize symmetric and theoretic 2D image-based non-contact anthropometric ideas, and to solve the problem of measuring body circumference dimensions on 2D images in clothing e-commerce. Firstly it separates 2D image-based non-contact Anthropometry into three steps: image silhouette extracting and characteristic body measurement, girth calculation and error \& accuracy analysis, and analyses the methods of every step; then it discusses the characteristics of the anthropometry operating and its' application in clothing e-commerce.
\end{abstract}

Key words: Non-contact, Anthropometry, 2D image, Clothing e-commerce.

\section{Introduction}

No two people are ever alike in all of their measurable characteristics; the first individual to research the beginning of anthropometry was Quelet in 1870, with his desire to obtain average measurements of male' every part [1]. Human body is the base of clothing structure design, only on the basis of accurate measurement of body dimensions, become design and manufacture of clothing possible, so anthropometry is the foundation of reasonable clothing structure design. Anthropometric methods include contact and non-contact anthropometry. Contact anthropometric method can be divided into Martin, sliding gauge, and replica; non-contact anthropometry can be divided into photogrammetry, moiré topography and 3D anthropometry [2]. The thesis breaks down 2D image-based non-contact Anthropometric technology into three steps, and analyses adopted method of every step and existing problem, then discusses anthropometric application to clothing e-commerce.

Non-contact Anthropometric technology was developed from the 1980's, a series of non-contact Anthropometric system have been developed successively by clothing industry developed countries---The United States, Britain, Canada, mainly there are 2D and 3D measurement method. 3D anthropometric system is high accurate, but it is expansive, enormous, measuring in darkroom, and equipment not moved conveniently, so the actual production application is difficult. Related scholars have launched 2D image-based anthropometric research in order to gain a practical and simple measurement technology. The basic idea is to use cameras produced positive and lateral direction of human body of 2D images (Fig.1); according to the processes of human body image denoising, edge detection, and contour extraction obtaining clear body contour images; Measuring each part of the width, thickness and length size, etc of body contour image; then by the curve fitting, regression analysis or grey relation analysis methods getting the human body circumference measurement. 

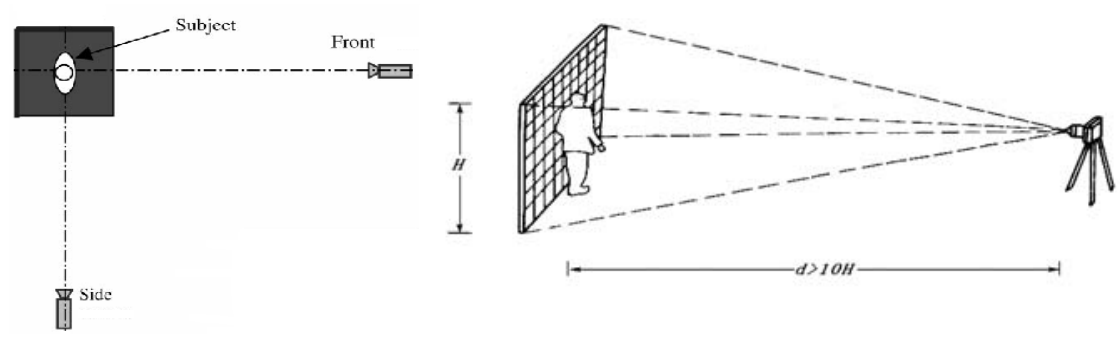

Fig.1. photographic methods

\section{Methodology}

\section{Human body contour extraction}

Before measuring hip width, hip depth, waist width, waist depth, etc sizes of feature points, human body contour processing can reduce error and improve anthropometric accuracy. Applying the principle of edge detection for digital image and three type algorithms based on the edge detective operators extracted 2D image edge. Through the comparison of the operators' actual effect, the result of experiments shows that Sobel and Smoothed operators have good effects on image enhancement, suppressing the noise and providing clear-cut profile lines, in favor of contour track and repair [3]. Based on the digital image edge segmentation principle of the threshold value ruled out internal and external factors on interference to human digital image; secondly, by analyzing points and lines feature of body digital image, Summarized five contour measurement methods in view of the different human body parts: curvilinear-knaggy-point recognition, extremum recognition, modeling recognition, curve line fitted recognition and approximate arithmetic of curve length [4]. Using MATLAB tools and computer graphics processing knowledge got body edge from ordinary digital photos, and combining the laws of the human body shape characteristics, and determined the feature points on the edge of body image [5]. Camera got images containing the information of human body characteristic line, 3D dimension of the human body was gained by more than two images. The steps were Firstly carrying on image format transformation, edge detection and so on a series of pretreatment, and extracting a two-dimensional size of chest, waist and hips in contour images [6]. Analyzed many commonly used algorithm of edge detection and contour extraction, with taking human body structure traits into account, a processing flow was designed involving image denoising, edge detection, contour extraction, measurement vertex search and coordinates computation, which can realize extracting body measurement automatically [7]. Algorithm was proposed with CANNY operator for edge detection extracting the edge of body figure, considering the abnormality eliminating accompanying with self-calibration, the measurement precision was improved with simplified manipulation [8].

When camera shoot images, subject slightly shake or camera jolt would lead to a blurry image edge. Camera technology promotion is the fundamental method to solve this problem, such as Sony's new research and development of the Exmor CMOS image sensors effectively resolve the blurry image edge. Before measuring silhouette feature position sizes, a succession of clear contour images are the foundation of accurate human body measurement, among them the key problem is how to choose appropriate method of image edge detection and properly determine the feature points.

\section{Circumference calculation}

\section{Curve fitting}

From the digital image it has access to 2D dimensions of the human body height, width and the thickness directly, comparing three function curves fitting precision, and finally determined that use 
logarithm function to fit age from 18 to 26 typical hip curves [9]. Functional model was established that circumferences related with height, weight, width, and thickness to estimate the circumferences. And an approach was presented taking advantage of samples' circumference curve to build human body truck model [10]. Double elliptic curve fitting model was employed to predict main circumferences, shoulder breadth, cervical height, and etc [5]. Exploring elliptical model fitting circumferences of the related positions on limbs identified the model suitable for fitting thigh circumference and calf circumference [11].

A different body position to adopt curve fitting models is different. To determine the most suitable fitting curve, we need to employ several fitting curve preliminarily selected, and compare their fitting accuracy, then choose a fitting curve with highest fitting accuracy as the fitting curve model for some position.

Regression analysis

Defined TNF index, hip width, hip depth, body front projection area and body side projection area as major parts for hip regression model, and the model was presented predicting hip size [12]. Least squares regression and partial least squares regression methods were introduced to measure bust circumference [13]. By means of correlation analysis and regression analysis, etc statistical analysis method was derived for bust regression formula for young women [14]. Using 2-stage least-squares analysis, linear regression analysis extracted two dimensional sizes of the human body height, width and the thickness, and then modeled to predict main circumferences, shoulder breadth, cervical height, and etc [5]. Linear regression were employed establishing circumference algorithmic model which take circumference value by manual measurement as dependent variable, and take depth and width value extracted automatically by programming as independent variable [15]. The photos were taken from the front, side and back of body, and these unorganized range data were used to conduct the body surface fitting. The body dimension was calculated by regression equations [16]. Least squares regression analysis method was presented which is suitable for calculating knee circumference and other major positions on limbs [11].

Regression analysis model have many types, every body part should apply multiple appropriate regression mathematical model to calculate, then through comparing calculation values with practical measurement value, can identified most appropriate regression model. Researches above are isolated; have not form a systematic theory. Regression analysis method is based on dimensions of other body parts to build relevant mathematical model and obtain circumference. Precision of the measurement method have significant relationship with sample number and shape similarity degree among samples, so there are dramatic error in this method.

Gray correlation analysis

By the application for the theory of gray correlation analysis, height, weight, bust width and bust depth were determined to the dominating body parts of gray model of bust circumference, and according to the classification of TNF index, the grey models of bust circumference are established [17]. Because human body is complex and changeable, each place are related to each other, these are in line with the characteristics of gray system, so can use gray model to measure circumference.

\section{Error and precision analysis}

Accordingly, the simple multiple linear regression model of hip girth is put forward, and its multi-colinearity is detected. In order to improve the regression precision, the independent variable is further selected using the stepwise regression method. At last, the ridge regression method is introduced to solve the multi-colinearity, which gives very good predicted results with an average error of 0.52 centimeters [12]. Increasing the experiment times and samples were proposed to improve the accuracy of experimental data and circumference model can be optimized by raising 
human body data to reduce error [16]. It can be concluded that the factors affecting the accuracy of human body photogrammetry include the optical constitution of the camera, manual operation, and subsequent measurement etc. Optical error is basically conformed to the law of spherical surface deformation. According to the analysis results the suggestion to choose suitable digital camera and optimize the measurement software for getting good results was brought forward. The error of combination Nikon D100 with Shapeline is least; combination of Canon PowerShot S400 and Photoshop got the error of the maximum degree [18]. Human body's characteristic dimensions were obtained by taking photos, reference calibration method was applied to gain measurements and the error was analyzed. Employing MATLAB programming image distortion degree can be lowered in order to reduce error, the high-contrast shooting background were presented to minimize error [19].

The reason causing error is more, from photo shooting, contour edge extraction to circumference calculation during every process different degree error will be generated, how to control error in a reasonable range need a further research.

\section{Discussion}

A database of 349 subjects (male and female) were measured not only with traditional anthropometric tools to gain data, but also with 2D image-based anthropometric measurement system taking simultaneous front and side pictures of individuals standing with their arms straight and slightly abducted along their side to collect images, then established mathematical model calculating. It was concluded no significant different between two data deriving from different methods both in terms of accuracy and repeatability [20]. an image-based system was proposed to obtain anthropometric measurements from photographic images more efficiently in e-commerce. Linear and circumferential measurements can be obtained within a certain accuracy and reliability [21]. Based on the clothing of e-commerce human body size needs much higher efficiency and convenience, a means was presented to obtain anthropometric measurements from photographic images. Among the traditional measurement and photographic measurement methods were compared, it was concluded that photographic measurement had very high precision and reliability [22].

Image-based measurements anthropometric measurements and traditional measurements were obtained directly from five athletic performers. Traditional measurements and image-based measurements were used in Yeadon's (1990) inertia model. The mean absolute accuracy in predicted whole-body mass achieved using traditional measurements and image-based approach was $2.10 \%$ and $2.87 \%$, respectively [23]. Anthropometric dimensions of the human body from calibrated monocular sequences, it focuses on estimating stature and shoulder breadth. Precise average estimates are obtained for each anthropometry by combining measurements from multiple frames in the sequence. The contribution is two-fold: a novel technique for automatic and passive estimation of shoulder breadth that is based on modeling the shoulders as an ellipse; and a novel method for increasing the accuracy of the mean estimates of both anthropometries. The latter is based on the observation that major sources of error in the measurements are landmark localization the 2D image and 3D modeling error [24]. A systematic method was presented to detect feature points on contour of human body from front and side images. Based on chain codes algorithm 55 feature points and 26 body measurements be extracted automatically. The method has been tested on four human subjects and all were correctly extracted [25].

Researchers mainly focus on contrast of artificial measurement and $2 \mathrm{~d}$ image-based measurement value. 2D image-based anthropometric measurement system takes simultaneous front and side pictures of individuals, the method can reduce the image measurement of synchronization 
and errors; Scholars put forward the method can be used to improve human body data collection efficiency in clothing e-commerce, but no specific discuss a key problem that is how to acquaint images simply and accurately. Because the actual application has error influence factors more than laboratory and controllability is poorer in practice.

\section{Conclusion}

Circumference calculation of 2D image-based non-contact anthropometric method include curve fitting, regression equation, graph mathematic model, gray correlation analysis, etc. the curve fitting model is diverse, but researches above are against young people, some region, or a particular shape, the universality of the model is not high, the law in view of the different shape and different body parts of the human body model don't discuss. Regression analysis method in the choice of measurement parts includes the height, weight, hip depth, hip width, waist depth, waist width and so on. Regression analysis accuracy is not stable which is related with sample number and sample discrete levels. Some scholars failed to take into account exact extraction of image contour, before curve fitting and regression analysis of circumference measurement, and the best way to deal with image contour is further confirmed, whether or not images contour is properly handled is the main reasons of causing errors.

A further investigation for 2D image-based non-contact anthropometric method needs to focus on operation simplicity, error precision, and etc. 2D image-based non-contact anthropometry is promising in clothing MTM online, at present clothing MTM online failed to solve the main problem is remote accurate acquisition of human dimension, the problem is bottleneck restricting development of clothing MTM online.

\section{Reference}

[1] Karla P. Simmons, Cynthia L. Istook. Body measurement techniques: comparing 3d body-scanning and anthropometric methods for apparel applications, Journal Of Fashion Marketing And Management, 7(3)(2003), p. 306-332.

[2] Fang Fang And Zhang Weiyuan. A new measurement technology: 3d body scanner system, China Textile Leader, 2 (2003), p. 34-37.

[3] Li Xiaojiu, Zhao Jingmiao, Wang Yuxiu. Application Of Edge Detection Methods Based On Operators To The Analysis Of Body Digital Images, Journal Of Textile Research, 3(27) (2007), p. 43-46.

[4] Zhao Jingmiao. Research on non-contact 3d body measurement system, Master Dissertation, (2004).

[5] Huang Xiuli. Research on measurement of young female's bodies based on digital images, Master Dissertation, (2009).

[6] Zhai Wenbin. Measurement of the human body based on image processing, Master Dissertation, (2006).

[7] Du Yanhua, Yang Zhiqiang. Research on automatic information extraction of human body based on photos, Computer Technology And Development, 2(20)(2010), p. 48-51.

[8] Yuan Yuan. Non-contact human body measuring technology based on camera self-calibration technique, Master Dissertation, (2005). 
[9] Li Xiaojiu, Wang Yuxiu. Hip calculation of 2d non-contact anthropometry system, Journal of Textile Research, 2(25)(2004), p. 98-100.

[10] Lu Cheng. The study and implementation of image-based non-contacting human body auto-measurement system, Master Dissertation, (2009).

[11] Qian Qian, Huang Xiuli,Liu Guolian. Research on prediction methods of limbs' girths of young females, Journal of Suzhou University( Eengineering Science Edition), 6(28)(2008), p. 24-28.

[12] Wang Yuxiu, Li Xiaojiu. Establishment of the ridge regression model of human hip girth, Knitting Industries, 7(2006), p. 27-29.

[13] Jiang An, Xu Zengpu, Yu Demin, Wang Yongqiang. Fitting methods for sizes of human bodies bust, Journal of Tianjin Polytechnic University, 5(21)(2002), p. 85-88.

[14] Xu Feng, Zhang Hao, Zheng Rong. Prediction for bust girth using regression analysis from photographic and anthropometric data, Journal Of Textile Research, 8(27)(2006), p. 49-52.

[15] Tan Fei. The research of youth female somatotype\&2D non-contact nomatometry system based on digital pictures, Master Dissertation, (2010).

[16] Zhu Fangfang, Luo Ronglei. 3d body measurement systems to the apparel from photos based on unorganized range data, Journal Of Zhejiang Sci-Tech University, 5(28)(2011), p. 710-713.

[17] Li Xiaojiu, Zhu Guangzhou. Establishment of grey models of human bust, Journal Of Textile Research, 2005,5(26), p. 77-79.

[18] Guo Jing, Zhang Hao, Zheng Rong, Hu Xiaodan, Duan Xiaojuan. Analysis on the factors influencing the accuracy of human photogrammetry, Journal Of Textile Research, 6(27)(2006), p. 51-54.

[19] Cai Jian , Lin Dajun , Guo Yanlong, Hao Meiqin. Feasibility study of obtaining human body’s characteristic dimensions based on the photo measurement, Journal Of Donghua University( Natural Science), 4(37) (2011), p. 481-517.

[20] Meunier P., Yin S. Performance of a 2d image-based anthropometric measurement and clothing sizing system, Applied Ergonomics, 31(5) (2000), p. 445-451 .

[21] Patrick Chi-Yuen Hung, Channa P. Witana, And Ravindra S. Goonetilleke. Anthropometric measurements from photographic images, Human Performance Laboratory, Department Of Industrial Engineering And Engineering Management, Hong Kong University Of Science And Technology, Clear Water Bay, Hong Kong,(2004), p. 764-769.

[22] Herianto, Silviana, Probandari And Agus Darmawan. Development of digital anthropometric circumferential measurement system based on two dimensional images, The 11th Asia Pacific Industrial Engineering And Management Systems Conference The 14th Asia Pacific Regional Meeting Of International Foundation For Production Research Melaka, (2010), p. 1-5.

[23] Marianne Gittoes, Ian Bezodis, Cassie Wilson. An image-based approach to obtaining anthropometric measurements for athlete-specific inertia modeling, 26 International Conference On Biomechanics In Sports (2008), p. 85-88. 
[24] Chiraz, Ben Abdelkader. Estimation of anthropometry from a single calibrated camera, Seventh IEEE International Conference On Automatic Face And Gesture Recognition (FG'06)(2006).

[25] Yueh-Ling Lin, Wang, M.-J.J. Automatic feature extraction from front and side images industrial engineering and engineering management, IEEE International Conference On Date:8-11 Dec. (2008), p. 1994-1953. 